\title{
Optimal Signal Timing Method of Intersections Based on Bus Priority
}

\author{
Junyou Zhang, Pengfei Li*, Yuyue Ma \\ College of Transportation, Shandong University of Science and Technology, Qingdao, China
}

\section{Email address:}

junyouzhang@sdust.edu.cn (Junyou Zhang),437361148@qq.com (Pengfei Li),534276015@qq.com (Yuyue Ma)

${ }^{*}$ Corresponding author

\section{To cite this article:}

Junyou Zhang, Pengfei Li, Yuyue Ma. Optimal Signal Timing Method of Intersections Based on Bus Priority. American Journal of Traffic and Transportation Engineering. Vol. 3, No. 1, 2018, pp. 1-5. doi: 10.11648/j.ajtte.20180301.11

Received: February 22, 2018; Accepted: March 11, 2018; Published: April 4, 2018

\begin{abstract}
According to the traffic problems such as traffic congestion and buses moving slowly, the paper based on the idea of bus priority to introduced the per capita minimum delay of signal control method. In this paper, the intersection of Huaguang Road and Liuquan Road in Zibo Zhangdian was used as an example to propose a bus signal timing plan. By considering the impact of traffic delays and designing the phase of the intersection. It can reduce the per capita delay and improve service levels. Using Trans Modeler to simulate the timing scheme, the results showed that the optimized scheme can effectively reduce the per capita delay and ensure priority access for buses.
\end{abstract}

Keywords: Signal Timing, Bus Priority, Average Delay, Phase Design, Traffic Simulation

\section{Introduction}

Along with the rapid development of urbanization, the structure of road network is more and more complex. As the bottleneck of traffic development, intersection delay becomes the key factor that affects the efficiency of urban road traffic. The survey found that a reasonable signal control scheme could help ease congestion on the roads. At present, the traditional intersection timing method takes the minimum of vehicle delay as the target to determine the signal period length of the intersection, and the green time ratio of the intersection is divided according to the phase traffic flow ratio. This kind of timing method treats the bus as the same as other social vehicles, does not consider the size of the passenger volume of all kinds of vehicles, and loses the social fairness [1]. If the right of bus priority is not guaranteed, the traffic delay at the intersection will increase, which will hardly reflect the efficient, safe and fast performance of public transport [2].

The signal control based on bus priority gives the bus the right of priority at the intersection. In general, bus priority control can be divided into two categories [3]: reducing bus delay and reducing per capita delay according to the different methods and strategies. This article focuses on how to reduce the per capita delay at intersections. Considering the large number of buses carrying passengers, the green time ratio of each phase of intersections is reasonably allocated according to the proportions of traffic volume, and priority is given to bus passing. This method is used to ensure the distribution of the phase flow of the large proportion of the bus to the larger green time ratio. This signal timing method can ensure bus priority, reduce vehicle delays at intersections and improve the operational efficiency of road traffic. By constructing the Trans Modeler micro simulation platform to simulate the operation of vehicles, comparing the traffic delays before and after signal timing optimization at intersections, providing the virtual environment and technical support for verifying the feasibility of the scheme, and providing decision-making basis for traffic managers [4-5].

\section{Traditional Signal Timing Scheme}

The main design parameters of intersection signal timing are signal period and green time ratio. The traditional signal timing scheme in the phase design more use of British scholar Webster's method to determine the parameters and calculate the vehicle delay, that is, under the specified conditions, the calculated total vehicle delay is the smallest to get the optimal solution [6-7]. The timing scheme does not distinguish between the bus and the social vehicles. The length of the 
signal period and the time of the green light depend on the traffic of the key flow direction. If the traffic flow and passenger flow are the largest in a certain phase, then this method is feasible. But in many cases, the maximum flow of traffic does not necessarily have the largest passenger flow. If there are more buses in the phase flow direction with a small traffic volume, it is not reasonable to use this method for traffic signal timing. It does not consider the factors of per capita delay, so it will be difficult to play the efficient and fast characteristics of public transportation.

In order to calculate the vehicle delay of the intersection, it is necessary to determine the parameters of the signal period and the green time ratio. Based on research and experimentation, the optimal signal period that minimizes the total delay of vehicles passing an intersection is given by:

$$
\begin{gathered}
c=\frac{1.5 L+5}{1-Y} \\
Y=\sum_{i=1}^{n} y_{i}=\sum_{i=1}^{n}\left(\frac{v}{s}\right)_{i}
\end{gathered}
$$

Where: $c=$ Intersection signal period;

$L=$ The total loss time of the signal period;

$\mathrm{Y}=$ The total traffic flow ratio across the intersection;

$y_{i}=$ Intersection of each phase flow;

$\nu=$ The actual traffic flow of the signal phase;

$S=$ Saturation flow rate of the phase;

$n=$ The number of key phases;

The green time ratio of each phase is proportional to the ratio of the flow rate of the phase, and the formula for the green time ratio is as follows:

$$
\lambda=\frac{c-L}{c} \times \frac{y_{i}}{Y}
$$

Where: $\lambda=$ Phase green time ratio at intersection; Obtain parking delays based on Webster formula:

$$
d=\frac{c(1-\lambda)^{2}}{2(1-q / S)}+\frac{(q / Q)^{2}}{2 q(1-q / Q)}-0.65\left(c / q^{2}\right)^{1 / 3}\left(c / q^{2}\right)^{(2+5 \lambda)}
$$

Where: $d=$ Average delay per car; $q=$ Arrival rate of the vehicle; $Q=$ Traffic in each phase

\section{Intersection Phase Design Based on the Smallest Per Capita Delay}

Bus signal priority means that without affecting the operation of the entire intersection or the main line of vehicles on the premise, using reasonable changes in phase order, phase time, insert special phase and other methods to ensure bus rapid transit through the intersection. According to the different research goals, the bus signal priority is divided into two kinds, one is to reduce bus delay as the target, and one to reduce the per capita delay [8-9].

The bus priority timing method based on the minimum per capita delay is aimed at reducing the overall delay of the traffic in all directions. In the process of optimization of signal timing by adjusting the signal phase and the phase of the time to give the bus priority [10]. In this paper, the traffic flow and the volume of passenger flow at the intersection are compared. On this basis, the maximum volume of passenger flow direction is given priority. By sorting the passenger volume, we can distinguish buses with higher carrying capacity than other vehicles. In order to avoid the queuing of vehicles at intersection, we should also consider the direction of traffic with larger traffic volume. In addition, the right turn vehicle does not conflict with other vehicles, and the effect of the right turn vehicle can not be considered when the phase is designed [11].

In this paper, the intersection of Huaguang Road and Liuquan Road in Zhangdian District of Zibo is taken as the research object. Based on the bus priority concept, the phase of intersection is adjusted according to the size of passenger flow to explore the appropriate signal timing scheme. In order to explain the phase design process, take Huaguang Road and Liuquan Road intersection as an example to make a detailed analysis, as shown in Figure 1, where the number indicates the direction of the vehicle. Suppose the passenger flow and the traffic flow meet the following relationship:

traffic flow: $8>2>5>11>1>4>10>7$

passenger flow : $8>5>2>11>4>10>1>7$

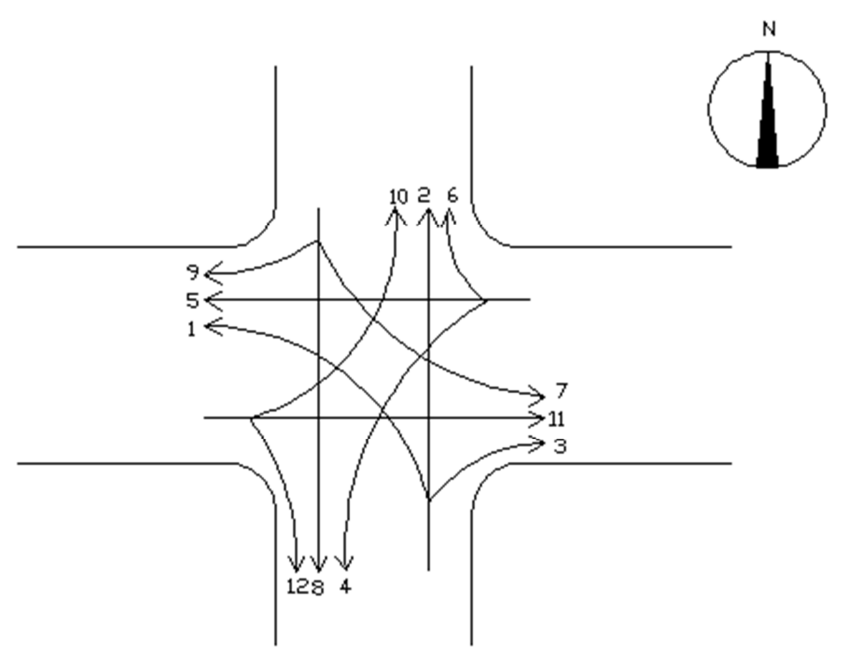

Figure 1. Huaguang Road and Liuquan Road intersection.

\subsection{General Phase Design Scheme}

Considering the basic principle of phase design and the condition of the traffic flow ratio, the phase design scheme 1 can be obtained, as shown in Figure 2. 


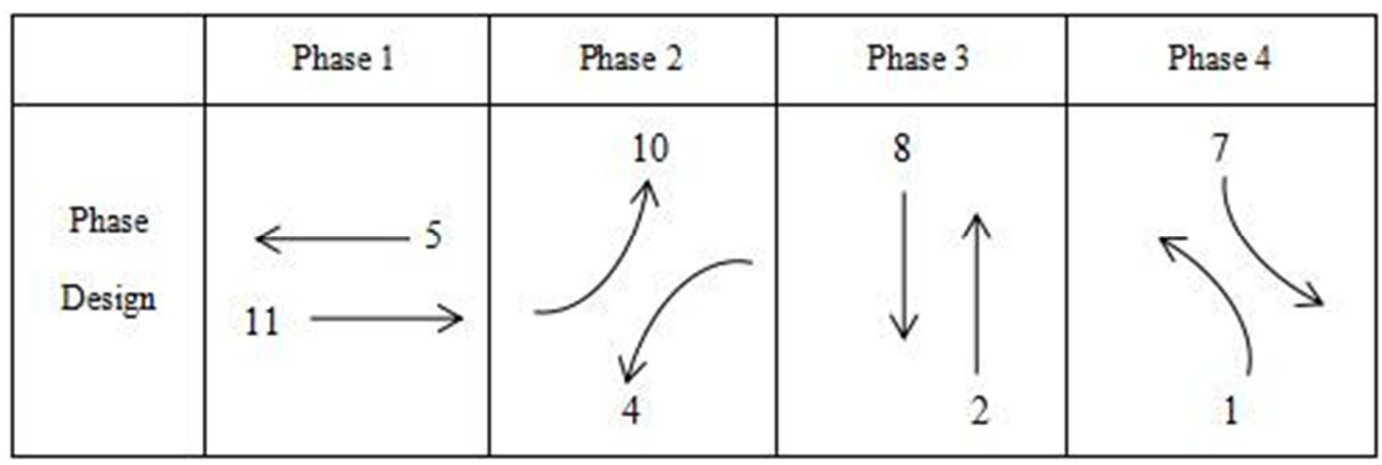

Figure 2. Phase design 1.

\subsection{Phase Design Scheme Based on Bus Priority}

Step 1: First of all, select the flow direction than the maximum passenger flow 8 , without considering the impact of non-motor vehicles and pedestrians, the left-turn and straight-ahead flows that do not conflict with this flow direction are $2,4,7$. So the combination that can be placed in the same phase as 8 is: 8 and 2, 8 and 4, 8 and 7. Taking into account the phase setting of the intersection should minimize the delay of social vehicles, select the combination of the largest traffic flow: 8 and 2 .
Step 2: Among the remaining flows, the traffic flowing to 5 is the largest, and the flows that do not conflict with this flow are 1, 4 and 11. The flow of traffic to flow 11 is the largest, choosing combinations 5 and 11 as the second phase.

Step 3: The flow direction that does not conflict with the flow direction 1 is $2,5,7$. Where 2,5 already belongs to the other phases, so that phase 3 is 1 and 7 . Finally get the phase 4 : 4 and 10. Phase design optimization scheme 2 shown in Figure. 3.

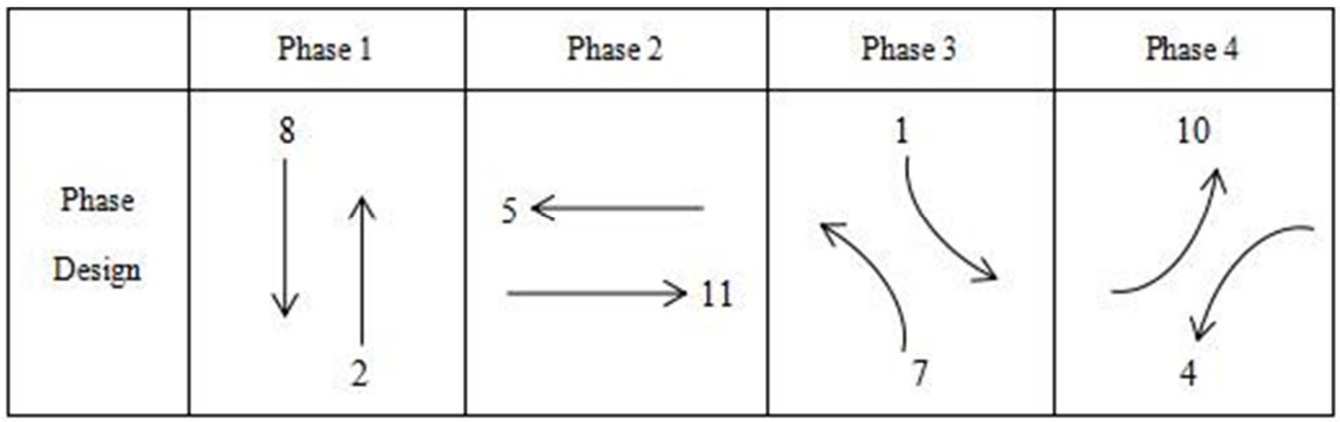

Figure 3. Phase design 2.

\section{Case Analysis}

\subsection{Basic Data}

Taking the intersection of Huaguang Road and Liuquan Road in Zhangdian District of Zibo City as the research object, the flow direction at the intersection is shown in Figure 1. In order to simplify the calculation, the effects of pedestrians and non-motor vehicles are not considered. Statistical data on the number of social vehicles and the number of buses at the intersection are shown in Table 1. Among them, the conversion coefficient of the bus is 2 , the average carrying coefficient of the car is 2.5 people / vehicle, the carrying coefficient of the bus is 30 people / vehicle, the single lane saturated traffic is $1800 \mathrm{pcu} / \mathrm{h}$.

Table 1. Vehicle flow and passenger flow of Huaguang and Liuquan intersection.

\begin{tabular}{|c|c|c|c|c|c|c|}
\hline Entrance & Direction & Social Vehicles (pcu/h) & Bus (veh/h) & Vehicle Flow (pcu/h) & Passenger Flow (people/h) & Flow Ratio \\
\hline \multirow{3}{*}{ North Entrance } & Turn Left & 394 & 0 & 394 & 985 & 0.22 \\
\hline & Straight & 610 & 20 & 650 & 2125 & 0.36 \\
\hline & Turn Right & 413 & 7 & 427 & 1242 & 0.24 \\
\hline \multirow{3}{*}{ South Entrance } & Turn Left & 344 & 10 & 364 & 1160 & 0.20 \\
\hline & Straight & 584 & 41 & 666 & 2690 & 0.37 \\
\hline & Turn Right & 405 & 9 & 423 & 1282 & 0.24 \\
\hline \multirow{2}{*}{ West Entrance } & Turn Left & 300 & 19 & 338 & 1320 & 0.19 \\
\hline & Turn Right & 502 & 28 & 558 & 2095 & 0.31 \\
\hline \multirow{3}{*}{ East Entrance } & Turn Left & 278 & 16 & 310 & 1175 & 0.17 \\
\hline & Straight & 558 & 16 & 590 & 1875 & 0.33 \\
\hline & Turn Right & 488 & 9 & 506 & 1490 & 0.28 \\
\hline
\end{tabular}




\subsection{Optimization Results Analysis}

According to the traffic survey and Webster's formula, the parameters of vehicle intersection delay and average delay at intersection are calculated, and the calculation results of delay are shown in Table 2.

Table 2. Delay compared on Huaguang and Liuquan intersection.

\begin{tabular}{llll}
\hline & Present Situation & Optimization & Percentage of Decline (\%) \\
\hline Vehicle Delay & 54.2 & 48.3 & 10.89 \\
Per Capita Delay & 52.8 & 44.5 & 15.72 \\
Vehicle Delay (Bus) & 43.1 & 36.8 & 14.62 \\
\hline
\end{tabular}

It is found through comparison that the timing scheme after phase change can not only reduce the average delay of vehicles at intersections, but also reduce the Per capita delay, which can ensure the transport benefits of buses and help to improve the traffic capacity and service level of intersections.

\subsection{Trans Modeler Simulation Verification}

This article takes the intersection of Huaguang Road and Liuquan Road in Zhangdian District of Zibo City as the object of simulation and carries out two simulations. The first simulated vehicle simulation did not use the bus priority signal timing scheme. The second simulation of the vehicle simulation, by changing the phase, added at the intersection of bus priority signal timing scheme. By comparing the results of two simulation outputs, such as the average delay and the average parking number to evaluate the feasibility of the optimization scheme [12-13].

The intersection model built with Trans Modeler simulation software is shown in Figure 4.

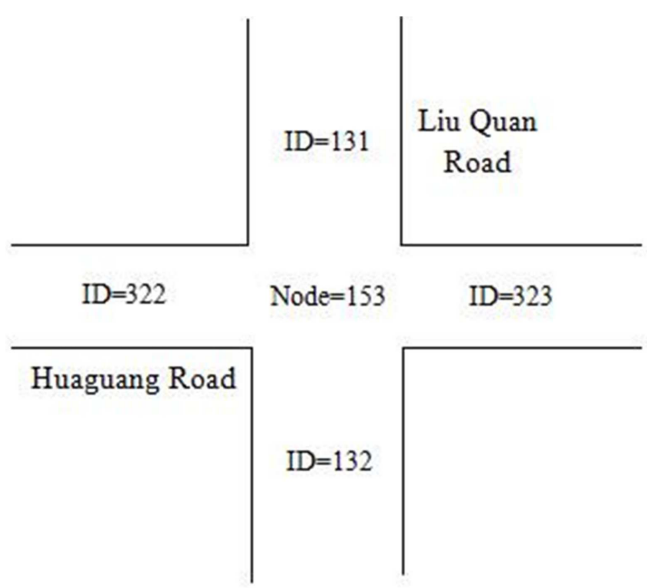

Figure 4. The road property of Huaguang and Liuquan intersection.

The simulation results before and after the implementation of the bus signal optimization scheme are compared as follows:

Table 3. Road delays analysis of bus signal optimization before and after.

\begin{tabular}{llllll}
\hline Evaluating Indicator & Timing Scheme & ID=131 & ID=132 & ID=322 & ID=323 \\
\hline \multirow{2}{*}{ Average Delay } & Pre Optimization & 40.2 & 33.1 & 105.1 & 44.8 \\
& After Optimization & 39.6 & 34.5 & 105.7 & 41.5 \\
Average Parking Time & Pre Optimization & 32.0 & 28.8 & 102.0 & 34.3 \\
& After Optimization & 31.6 & 28.2 & 102.6 & 31.3 \\
\multirow{2}{*}{ Average Parking Number } & Pre Optimization & 0.75 & 0.50 & 0.35 & 0.90 \\
& After Optimization & 0.70 & 0.55 & 0.40 & 0.80 \\
\hline
\end{tabular}

Table 4. Intersection delays analysis of bus signal optimization before and after.

\begin{tabular}{lllll}
\hline Intersection & Timing Scheme & Total Delay & Average Delay & Service Level \\
\hline \multirow{2}{*}{153} & Pre Optimization & 204120 & 58.6 & E \\
& After Optimization & 183240 & 43.3 & D \\
\hline
\end{tabular}

By comparing and analyzing the simulation data, it is concluded that after the implementation of the bus priority control scheme, the average delay at the intersection is significantly reduced, and the service level at the intersection is upgraded from the original $\mathrm{E}$ level to $\mathrm{D}$ level. The simulation results further verify the feasibility of the bus priority signal timing scheme. From the aspect of reducing per capita delay, the operation efficiency of the bus is improved, and the priority of public traffic is ensured.

\section{Conclusion}

The traditional signal timing method only considers the traffic flow ratio when designing the timing scheme and does not consider the passenger flow ratio. Although the vehicle delay can be minimized, the average per capita delay can not be guaranteed. In this paper, the traffic benefit indexes before and after phase change of bus priority signal timing scheme are analyzed through examples. The simulation of vehicle running status with TransModeler micro-simulation software is carried out to verify the feasibility of phase optimization scheme. The simulation results show that the scheme with the minimum delay per capita is not only able to guarantee the minimum per capita delay, but also reduce the vehicle delay and improve the service level at the intersection. 


\section{Acknowledgements}

This study was supported by The Scientific Research Foundation of Shandong University of Science and Technology for Recruited Talents (2015RCJJ035).

\section{References}

[1] Z. Junyou, L. Yaping. Research on Bus Priority Control on Non-coordinated Phase Based on Transmodeler —A Case Study of Changjiang Road in Huangdao District of Qingdao [J]. American Journal of Traffic and Transportation Engineering, 2017, 2 (3):32-38.

[2] L. Yang, Z. Shengchuan, Z. Jianwu. Delay analysis and signal control amelioration of intersection using bus priority signal-planning strategy [J]. Traffic Standardization, 2010. 1 (123):78-83.

[3] Y. Ronghan, W. Xiaoyu, X. Hongfeng, L. Lian. Emission factor calibration and signal timing optimisation for isolated intersections [J]. Iet Intelligent Transport Systems, 2018, 12 (2):158-167.

[4] J. Binbin, X. Jianmin. A General Maximum Progression Model to Concurrently Synchronize Left-Turn and through Traffic Flows on an Arterial [J]. Mathematical Problems In Engineering, 2018.

[5] L. Li, L. Yisheng, W. Feyue. Traffic Signal Timing J. Junchen, M. Xiaoliang. Hierarchical multi-agent control of traffic lights based on collective learning [J]. Engineering Applications Of Artificial Intelligence, 2018, 68:236-248.

[6] Y. Juan, L. Peizhong. Leaming traffic signal phase and timing information from low-sampling rate taxi GPS trajectories [J]. Knowledge-Based Systems, 2016, 110:275-292.

[7] Y. Quan, L Zhihan, L. Jinglin, Z. Junming, Y. Fangchun. A traffic congestion detection and information dissemination scheme for urban expressways using vehicular networks [J]. Transportation Research Part C-Emerging Technologies, 2014, 47:114-127.

[8] Z. Hantao, L. Xinru, C. Xiaoxu, L. Jiancheng. Cellular automata model for traffic flow at intersections in internet of vehicles [J]. Physica A-Statistical Mechanics And ITS Applications, 2018, 494:40-51.

[9] J. Junchen, M. Xiaoliang. Hierarchical multi-agent control of traffic lights based on collective learning [J]. Engineering Applications Of Artificial Intelligence, 2018, 68:236-248.

[10] R. Lanlan, Z. Yao, H. Haoqiu, Q. Xuesong. A new traffic congestion detection and quantification method based on comprehensive fuzzy assessment in nanet [J]. Ksii Transactions On Internet And Information Systems, 2018, 12 (1):41-60.

[11] Q. Liang, Z. MengChu, L. WenJing. A two-level traffic light control strategy for preventing incident-based urban traffic congestion [J]. Ieee Transactions On Intelligent Transportation Systems, 2018, 19 (1):13-24.

[12] O. Younis, N. Moayeri. Employing cyber-physical systems: dynamic traffic light control at road intersections [J]. Ieee Internet Of Things Journal, 2017, 4 (6):2286-2296.

[13] L. Zhiyi, M. Shahidehpour, S. Bahramirad, A. Khodaei. Optimizing traffic signal settings in smart cities [J]. Ieee Transactiongs On Smartgrid, 2017, 8 (5):2382-2393. 